\title{
Transient Thermal Phenomena during Spontaneous Water Migration in Zeolite Beds
}

\author{
J. ŻoŁĄDEK-NowAK*, J.J. MilczAREK, I.M. FiJAŁ-KiREJCZYK, J. ŻoŁĄDEK \\ AND Z. JURKOWSKI \\ National Centre for Nuclear Research, A. Sołtana 7, 05-400 Otwock-Świerk, Poland
}

\begin{abstract}
The changes in local temperature occurring during spontaneous imbibition of columnar samples of dry natural zeolite with water were studied. The temperature pulses of $20-250 \mathrm{~s}$ duration and $3-40^{\circ} \mathrm{C}$ amplitude were registered and correlated with the wetting front motion observed with neutron radiography. The amplitude of the pulses was shown to increase with increasing ambient temperature. The broadening of the temperature pulse with increasing distance from the water supplied end of the samples was observed. The amplitude and the pulse width were found to depend substantially on the drying temperature of the material. The observed effect was attributed to the heat of immersion released in the sample at the moving wetting front.
\end{abstract}

PACS: 82.75.Jn, 61.05.Tv, 47.56.+r, 81.05.Rm

\section{Introduction}

The most spectacular phenomenon observed in the liquid migration in porous system is the moving wetting front due to spontaneous imbibition of the porous medium with wetting liquid. That motion of the wetting front in unsaturated (i.e. dry) porous medium is inseparable from the wetting process itself which is driven by the reduction of the system free energy. The released surplus of free energy, named the heat of immersion [1], yields an increase in local temperature initiating local temperature spikes, a phenomenon that seems to have been forgotten and rediscovered many times [2,3]. It is obvious that a significant heat release should be expected during wetting of zeolites characterized with large specific surface. The vast absorption capacity of zeolites has been applied in various heating and cooling systems already $[4,5]$. The release of the immersion heat during wetting of a natural zeolite is envisaged as a basic effect for a kind of energy storage facility where solar energy would be used for drying of the zeolite and the immersion of the material in water would produce the usable heat [6].

The main objective of the present work was to obtain experimental data on the temperature spikes occurring during spontaneous capillary rise of water in unsaturated beds of natural zeolite (clinoptilolite). The fine grain material, for which no gravitational effects on the imbibition height was observed previously $[7,8]$, was chosen for the

\footnotetext{
* corresponding author; e-mail:

Joanna.Zoladek-Nowak@ncbj.gov.pl
}

study. In our previous work $[7,8]$ we found significant dependence of the rate of the spontaneous imbibition in clinoptilolite on ambient temperature as well as on the dryer temperature at which the material was dried before the wetting. In this work the dependence of local temperature pulses' parameters (amplitude and time width) on the ambient temperature of the wetting process and the temperature is investigated.

\section{Experimental}

The samples were prepared from natural zeolite containing $84 \%$ of clinoptilolite and composed of $0-0.2 \mathrm{~mm}$ sized grains. Before the experiment the material for the samples was rinsed with deionized water for $24 \mathrm{~h}$ and then dried for $2-5$ weeks at one of three drying temperatures $\left(T_{\mathrm{a}}\right) \approx 50, \approx 100$ or $\approx 150{ }^{\circ} \mathrm{C}$. The samples were prepared from the dried material as cylindrical columns contained in aluminum tubes of $7 \mathrm{~mm}$ inner diameter and $135 \mathrm{~mm}$ length. The samples were produced by pouring the dry powder into tubes and simultaneous short time (3 min) and small amplitude $(1 \mathrm{~mm})$ vibrations. The effective porosity of the samples was $\approx 0.55$.

Experiments were carried out at the dynamic neutron radiography facility NGRS at the nuclear research reactor MARIA [7, 9]. Dry samples were placed inside a thermal chamber (Fig. 1b) with double thermal shields $[7,10]$. The ambient temperature of the sample $\left(T_{\mathrm{s}}\right)$ was chosen to be $30,40,50,60$, or $70^{\circ} \mathrm{C}$ and was stabilized within $\pm 0.1^{\circ} \mathrm{C}$. Four K-type steel sheathed thermocouples of $0.5 \mathrm{~mm}$ diameter were inserted into each sample at different distances $\left(x_{1}=11.3, x_{2}=30.7, x_{3}=51.3\right.$, $x_{4}=101 \mathrm{~mm}$ ) from their lower end (Fig. 1a). The due 


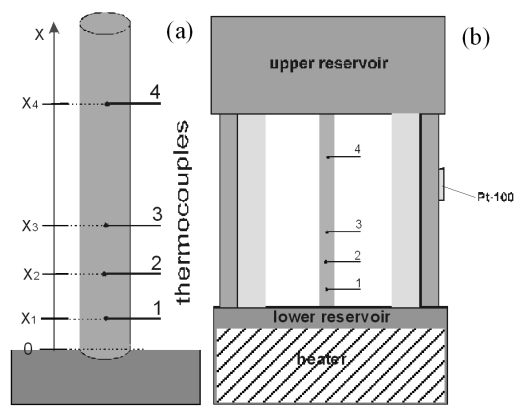

Fig. 1. The arrangement of thermocouples in the sample (a) and the position of the sample in the thermal chamber (b).

care was paid to place thermocouples' ends at the middle of the tube. When the ambient temperature got stable, water was introduced into the lower container just below the lower end of the sample. The thermocouples' readings and neutron radiographs were registered on-line by two separate computer systems. The moment of contact of water with lower end of the zeolite bed was defined from the recorded sequences of neutron radiographs and the wetting front position was determined as the distance of the representing water dark region boundary from the lower end of the sample [7-10].

\section{Results}

The pronounced temperature spikes were observed for all thermocouples and experimental conditions applied (Fig. 2). The temperature fluctuations in registered records were less than $0.1^{\circ} \mathrm{C}$ and completely insignificant. The pulses are clearly distinguishable from the small (less than $0.5^{\circ} \mathrm{C}$ ) and slowly relaxing (in $\approx 1000 \mathrm{~s}$ ) increase in the local temperature observed just after water touched the sample's end. The time duration of the spike increased from $\approx 20 \mathrm{~s}$ for the lowest thermocouple to $\approx 300 \mathrm{~s}$ for the highest one. The shape of the pulse depends substantially on the distance $x$ from the water supplied end of the sample. The initial steep front of the emerging pulse followed by rather long decay period observed at the first thermocouple turned into long rise and relatively shorter decay of the temperature transient found at the furthest thermocouple (Fig. 3).

The main characteristic parameters describing the pulses emerging at the thermocouples were determined from the recorded variations of the thermocouples' readings. The set of pulse parameters comprised the time $t_{M i}$ of occurrence of the pulse maximum at the $i$-th thermocouple $(i=1 \ldots 4)$, the time width $\Delta t_{i 1 / 2}$ at the half of its maximum, and the pulse amplitude defined as the difference between the temperature peak value $T_{M i}$ and the ambient temperature $T_{\mathrm{s}}$. The pulse amplitude was within $10-20^{\circ} \mathrm{C}$ for the first thermocouple and was approximately twice that observed for clay and fine grain quartz in experiments performed in the same arrangement. In most cases the temperature pulse amplitude
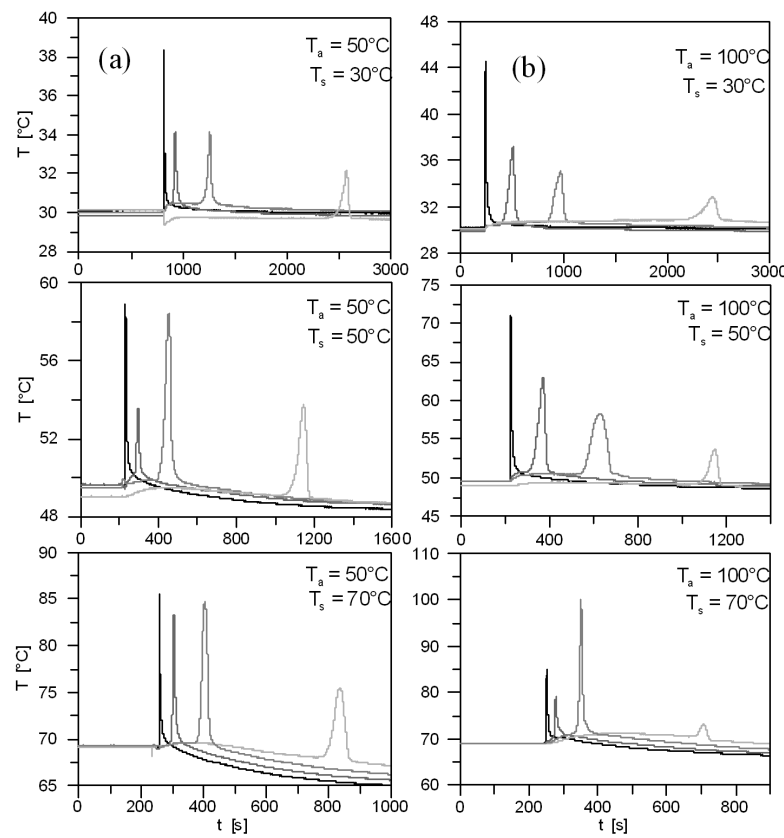

Fig. 2. Examples of the time dependence of local temperature at different ambient temperature for samples prepared from the material dried at $50{ }^{\circ} \mathrm{C}$ (a) and $100^{\circ} \mathrm{C}(\mathrm{b})$.

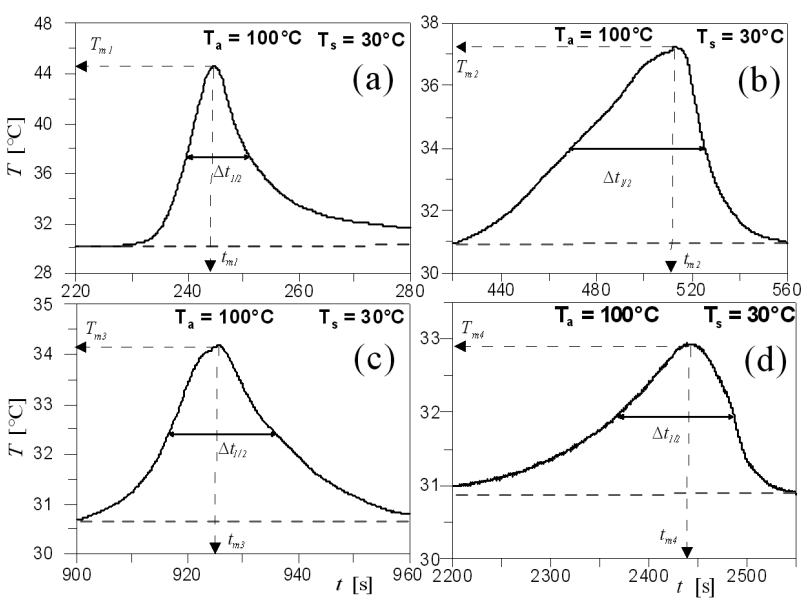

Fig. 3. Example of the local temperature time dependence at ambient temperature of $30^{\circ} \mathrm{C}$ for sample dried at $100{ }^{\circ} \mathrm{C}$. (a) Plot for the first thermocouple, (b) second, (c) third, and (d) for the fourth one.

was decreasing with the distance of the thermocouple from the water supplied end of the column. The increase of the pulse height with ambient temperature was observed on the average (Fig. 4). We should note that it reached even $40^{\circ} \mathrm{C}$ at first thermocouple for process carried out at $60^{\circ} \mathrm{C}$ and zeolite dried at $150^{\circ} \mathrm{C}$. However, neither the thermal contact between the medium and thermocouples' junctions was perfect, nor the thermocouples were isolated thermally from the aluminum 


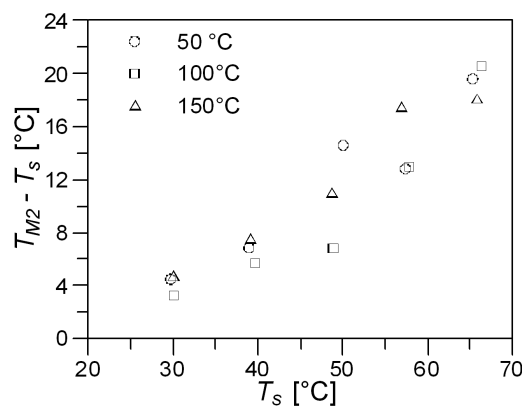

Fig. 4. Example of the dependence of the temperature pulse amplitude observed at the second thermocouple position on the ambient temperature.

tube wall. In effect, some deviations from the smooth unambiguous dependence of the pulse height on ambient temperature were observed (Fig. 4).

\section{Discussion}

We found that the temperature pulse became more diffuse and lower with the increasing distance $x$ from the lower end of the sample (Fig. 2). The pulse width increased substantially with that distance (Fig. 3). That increase in pulse width should be linear in $x$ according to the classical [9-11] square root of time dependence

$$
x=A \sqrt{t}
$$

of the wetting front distance from the water supplied end of the sample. In this case the wetting front linear velocity $v$ is inversely proportional to the wetting front distance

$$
v=A^{2} / 2 x .
$$

Assuming that the width $w$ of the region occupied by the thermocouple's head is small in comparison to the distance $x$ (Fig. 5) we get for the time $\tau$ needed for the wetting front to pass through that gauge region

$$
\tau=\left(2 w / A^{2}\right) x .
$$

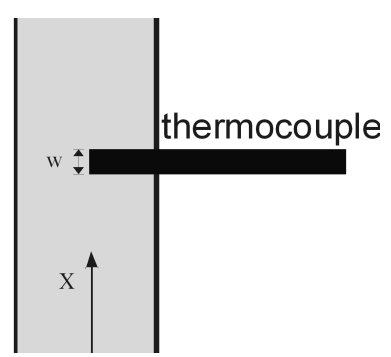

Fig. 5. Diagram of the active region of the thermocouple inserted into the sample.

Our results reveal that the linear dependence of the $\Delta t_{i 1 / 2}$ on $x_{i}$ was hardly observed (Fig. 6). However, one should note that the time width of temperature pulse is significantly larger (3 to 5 times) for samples dried at

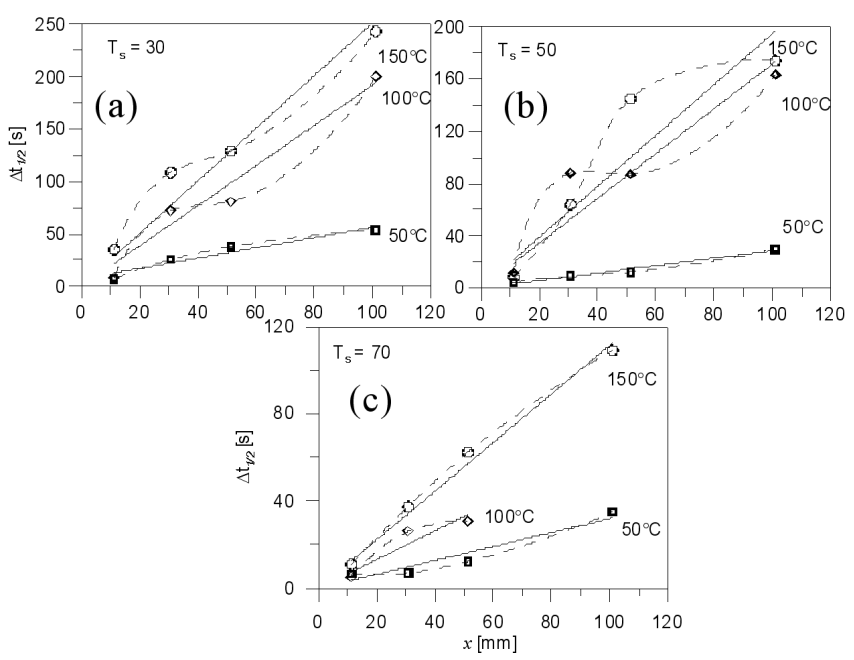

Fig. 6. Examples of the dependence of the width (FWHM) of the temperature pulse on the distance from the water supplied end of the sample for $30{ }^{\circ} \mathrm{C}(\mathrm{a}), 50{ }^{\circ} \mathrm{C}$ (b) and $70^{\circ} \mathrm{C}$ (c). The solid lines are linear fits and the broken ones are spline fits.

temperature at 100 or $150{ }^{\circ} \mathrm{C}$ than for $50^{\circ} \mathrm{C}$ dried specimens (Fig. 6). This effect is due to low water migration rate observed previously in 100 or $150^{\circ} \mathrm{C}$ dried material [8]. The wetting rate coefficient $A$ for material dried at 100 or $150^{\circ} \mathrm{C}$ is approximately five times smaller than for clinoptilolite dried at $50^{\circ} \mathrm{C}$. That decline of the water migration rate with higher drying temperature can be attributed to reduction of the water film covering the pores' boundary surfaces by enhanced evaporation during drying at higher temperatures.

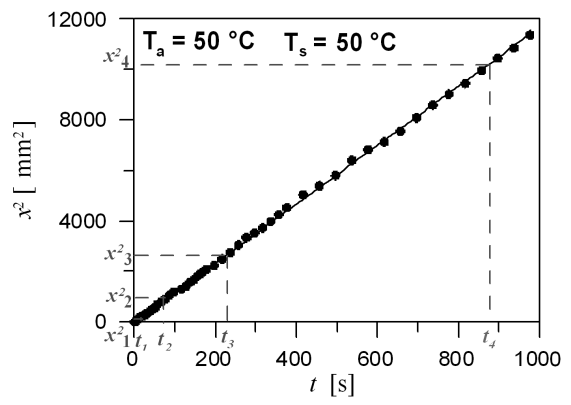

Fig. 7. Example of the time dependence of the wetting front position. The thermocouples' coordinates and corresponding times of front passage are marked with broken lines.

The times $t_{f i}(i=1 \ldots 4)$ of the wetting front passage over each thermocouple position $\left(x_{i}\right)$ was determined from the time dependence of wetting front position and known arrangement of thermocouples (Fig. 7). In order to check whether the moment of the maximum temperature occurrence $\left(t_{M i}\right)$ at given position within the sample coincided with the moment of wetting front passage over that position, we determined the relative differences 


$$
\Delta t_{i} / t_{f i}=\left(t_{f i}-t_{M i}\right) / t_{f i}
$$

between the time of front passage and time of the maximum temperature occurrence. Since the times of both events were determined by separate computer systems, we assumed that their coincidence is perfect at the first thermocouple position i.e. $t_{f 1}-t_{M 1}=0$. The assumption is based on the sharpness of the temperature pulse observed at first thermocouple and very fast motion of the wetting front at the beginning of the process.

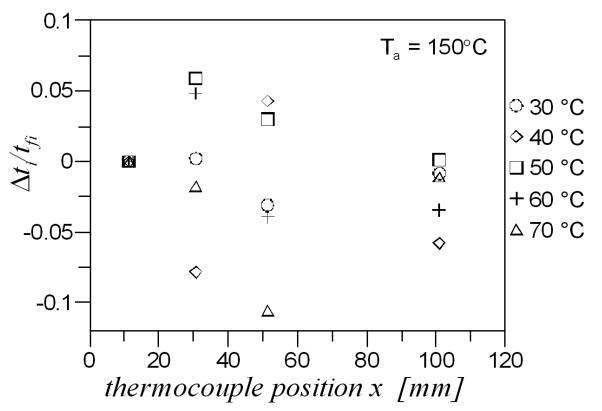

Fig. 8. Example of the relative differences between moments of wetting front passage and maximum temperature occurrence for three thermocouples for samples dried at $150{ }^{\circ} \mathrm{C}$. It was assumed that for the first thermocouple both effects coincide.

We found that the relative differences vary in sign and magnitude (Fig. 8) and apparently there is no strong statistical correlation between both times considered. However, the relative differences are quite small and their absolute values are smaller than the widths of the spikes at half maximum i.e.

$$
\left|\Delta t_{i}\right|<\Delta t_{i 1 / 2}
$$

suggesting that the moment of front passage can be identified with the occurrence of the temperature pulse although not necessarily with that of attainment of the maximum temperature.

The hypothesis that the temperature pulses are produced by release of the immersion heat is supported to be observed increase of the pulse amplitude with increasing ambient temperature. We found previously that the contact angle between water and the zeolite surface decreases substantially when the ambient temperature is increased reaching almost perfect wetting for temperatures higher than $60^{\circ} \mathrm{C}[8]$.

\section{Conclusions}

The temperature pulses emerging during water migration in particulate systems were observed for spontaneous migration of water in unsaturated beds of natural clinoptilolite.
The effect is attributed to the immersion heat of natural zeolite, which is released locally in the form of short pulses during the passage of the wetting front through the porous medium. The dissipation of the heat in the medium is responsible for the decay of the temperature after wetting front passage. The amplitude of the temperature pulses is higher in zeolite than in natural materials as clay or quartz grain beds observed in the same experimental arrangement. The amplitude and the time width of these pulses depend on the ambient temperature of the process and drying temperature of the material. The experimental data confirm that more heat is released during wetting of zeolite with reduced amount of water film covering the pores' boundary surfaces.

\section{References}

[1] D.M. Anderson, in: Methods in Soil Analysis. Part 1. Physical and Mineralogical Methods, Ed. A. Klute, American Society of Agronomy, Madison 1986, p. 969.

[2] D.M. Anderson, A. Linville, G. Sposito, Soil Sci. Soc. Am. Proc. 27, 610 (1963).

[3] L. Prunty, J. Bell, Soil Sci. Soc. Am. J. 69, 766 (2005).

[4] D.I. Tchernev, in: Natural Zeolites, Eds. L.B. Sands, F.A. Mumpton, Pergamon Press, Oxford 1978, p. 479.

[5] K.E. N'Tsoukpoe, H. Liu, N. Le Pierrés, L. Luo, Renew. Sustain. En. Rev. 13, 2385 (2009).

[6] H. Baoqi, et al. in: Integrated Energy Systems in China - The Cold Northeastern Region Experience, Eds. L. Nan, G. Best, C.C. de Carvalho Neto, FAO Rome, 1994, http://www.fao.org/docrep/T4470E/ t4470e00.HTM\#Contents.

[7] J. Żołądek, J.J. Milczarek, I. Fijał-Kirejczyk, Nukleonika 53, S113 (2008).

[8] J. Żołądek, J.J. Milczarek, I. Fijał-Kirejczyk, in: Topics in Chemistry and Material Science, Vol. 4, Eds. K. Hadjiivanov, V. Valtchev, S. Mintova, G. Vayssilov, Heron Press, Sofia 2010, p. 175.

[9] J.J. Milczarek, A. Czachor, A. El-Ghany El Abd, Z. Wiśniewski, Nucl. Instrum. Methods Phys. Res. A 542, 232 (2005).

[10] J.J. Milczarek, I. Fijał-Kirejczyk, J. Żołądek, M. Chojnowski, G. Kowalczyk, Acta Phys. Pol. A 113, 1245 (2008).

[11] E.W. Washburn, Phys. Rev. 17, 273, 374 (1921). 